\title{
Neoantigens from the bench to the bedside: new prospective for ovarian cancer immunotherapy
}

\author{
Chiara Napoletano ${ }^{1}$, Filippo Bellati ${ }^{2}$ \\ ${ }^{1}$ Department of Experimental Medicine, University of Rome "Sapienza”, Rome, Italy; ${ }^{2}$ Surgical and Medical Department of Translational Medicine, \\ Sant'Andrea Hospital, University of Rome "Sapienza”, Rome, Italy \\ Correspondence to: Chiara Napoletano, PhD. Laboratory of Tumor Immunology and Cell Therapy, Viale Regina Elena 324, 00161 Rome, Italy. \\ Email: Chiara.napoletano@uniroma1.it. \\ Provenance: This is an invited article commissioned by the Editorial Office, Annals of Translational Medicine. \\ Comment on: Liu S, Matsuzaki J, Wei L, et al. Efficient identification of neoantigen-specific T-cell responses in advanced human ovarian cancer. J \\ Immunother Cancer 2019;7:156.
}

Submitted Oct 10, 2019. Accepted for publication Oct 17, 2019.

doi: $10.21037 /$ atm.2019.10.62

View this article at: http://dx.doi.org/10.21037/atm.2019.10.62

Ovarian cancers (OCs) are considered today as immunogenic tumors. Several studies conducted on tumor infiltrate largely demonstrate that the presence of tumor infiltrated lymphocytes (TILs) correlates to progression (PFS) and overall survival (OS) in patients with advanced stage disease (1) and that the prognostic significance of TILs is an independent factor (2). $\mathrm{CD}^{+} \mathrm{T}$ cells seem to be primary actors in this scenario since their presence correlates with patients' survival in all stages and histologies (2-4). In addition, current therapeutic strategies have demonstrated to reduce immune suppression and increase cytotoxic response $(1,5)$. In the last decades, several new therapeutic strategies have been introduced and have demonstrated immunological advances in OC (6-8), but despite these improvements, over $60 \%$ of patients relapse and only $28 \%$ of patients survive after 5 years (9).

Due to the great impact that the immunological fitness has on the clinical outcome of OC patients, in the last two decades, several efforts were focused on the development of immunotherapeutic strategies, such as cancer vaccines, lymphocyte transfer and immunomodulatory therapy, to be administered alone or in combination with standard therapies (10). Although several studies have showed promising results in the generation of specific anti-tumor $\mathrm{T}$ cell response, this immunological activation/modulation is frequently not correlated to an evident clinical benefit $(11,12)$. This absence of success could be partially ascribed to insufficient patients' accrual or selection carried out in these analyses. There are rare studies conducted on women with OC primary tumor or in optimal immunological status, instead, analyses are carried out on women that are frequently selected by recurrent disease and/or heavy tumor burden. It is widely demonstrated that this immunosuppressive condition severely compromises the success rates.

Neoantigens represent today a new immunological weapon against tumor with the great potential to evoke a robust, prolonged and specific response against cancer cells. These molecules are highly immunogenic because they are not present in normal tissues and neoantigen specific $\mathrm{T}$ cells evade central tolerance (13). These immunogens can both expand pre-existing neoantigens-specific $\mathrm{T}$ cells and induce a broader repertoire of new $\mathrm{T}$ cell specificity in cancer patients and this can favour and enhance tumor control. In addition, patients with a clinical response to immune checkpoint inhibitors, recently introduced in the clinical setting of other neoplasms, show neoantigens specific $\mathrm{T}$ cells (14), suggesting that the identification of immunogenic neoantigens for adoptive $T$ cell therapy or for vaccination have the potential to have a clinical impact. Currently, among the high number of putative screened neoantigens in several solid tumors, less than 0.5 and $2 \%$ were validated as immunogenic.

Liu et al. (15) describe a new method to screen the immunogenic neoantigens from OC tissue that significantly improves the validation rate from $0.5-2 \%$ to $19 \%$ through 
in silico prioritization. These authors selected 20 OC patients (17/20 FIGO IIIC underwent to optimal debulking surgery) and performed whole-exome sequencing on pretherapy tumor and matched normal samples. Two thousand and ninety-six somatic mutations were identified including 1,368 non-synonymous somatic mutations. Candidate neoantigens-derived peptides were selected to have a higher affinity and specificity for patients' MHC compared to the corresponding wild-type. This strict peptides' selection was carried out to avoid the generation of neoepitope reactive TCRs that cross-react with both wild-type and mutate epitopes, limiting the elimination of T-cell precursors expressing such TCRs by the thymus. Four hundred and forty-nine neoantigens candidate were found to have a strong and specific binding affinity with MHC class I and/or MHC class II. These antigens were further classified considering the mutant allele's expression level in RNAseq data, but only half of them [209] showed a robust expression of the mutant allele.

Before peptide synthesis, neoantigens candidates were prioritized according to the following criteria: (I) mutation in cancer gene census (CGC) genes; (II) MHC binding affinity of the mutant allele; (III) different binding affinity between the mutant allele and the matched wild-type; (IV) variant allele fraction (VAF) of mutation; (V) expression level of the mutated allele and the overall level of the gene; (VI) type of MHC binding; (VII) biochemical properties related to peptide synthesizability.

Seventy-five neopeptides were selected (36 MHC I, 32 MHC II, 7 MHC I and II), 25 of them did not demonstrate to have a robust mutant allele expression. These 25 peptides were also included in the immunological evaluation to understand the relationship between low allele expression level and the induction of $\mathrm{T}$ cell response. The immunogenicity of selected peptides was analysed in those patients with blood and tumor specimens and the specific neoantigen $\mathrm{T}$ cells were characterized. PBLs and TILs of 10 patients were stimulated with specific neopeptides or with neopeptide pools. Twenty-seven IFN $\gamma$-producing $\mathrm{T}$ cells were detected in 5 patients out of $10,4 / 5$ patients showed an activation of both peripheral blood lymphocytes (PBLs) and TILs. Results demonstrated that: (I) as expected, patients with a specific neoantigens $T$ cells have a higher mutational burden compared to those without; (II) peptides with a low expression of mutated allele were able to induce a similar specific $T$ cell response compared to those peptides derived from mutated allele with a high expression, suggesting that a small amount of neoantigen with high avidity for TCR can elicit a strong immune response; (III) lymphocytes derived from tumor seemed to elicit a stronger $\mathrm{T}$ cell response than $\mathrm{T}$ cells isolated from blood (in 3 patients out of 4) and this imply that generally tumor specific lymphocytes can migrate into the tumor and that TILs not compromised by immunosuppressive signals could elicit a strong anti-tumor immunity; (IV) the inhibitory molecules analysed at RNA level in the 4 patients with both PBL and TIL response were higher in that patient that showed the strongest immune response against single neoepitopes and neoepitope pools, indicating that the activation induced by these type of antigens is strong and induce a "chronic inflammation"-like response; (V) responding patients had an enriched pathway related to processing and presentation machinery compared to nonresponding, suggesting that this complex machinery impact the type of immune response probably contributing to generate a new antigen repertoire against tumor.

Neoantigen reactive $T$ cells were further isolated from TILs of OC patients, expanded and established as neoantigens specific $\mathrm{T}$ cell lines. In particular, CD4 $\mathrm{T}$ cells reactive against mutated NUP214 and JAK1 were obtained. Low resolution TCR V $\beta$ spectratyping identified the response against NUP214 and JAK1 as oligoclonal and monoclonal, respectively. Both populations were high tumor specific, reacting only against autologous tumor, but not against PBMCs.

The TCRs of these cells were also transferred to $\mathrm{T}$ cells of healthy donors to test the feasibility to transfer the neoantigens specificity to other $\mathrm{T}$ cells. All the engineered lymphocytes showed a strong mutated peptide-specificity after 2 transductions.

The paper by Liu et al. has been carried out with a rigorous methodology and on an adequate sample of patients.

The identification of neo-antigens is extremely appealing since it is perfectly in line with the current clinical objectives of identifying strategies to carry personalized medicine and the introduction of novel drugs such as checkpoint inhibitors that are able to elicit and improve immune response of TILs. This approach has only recently been possible as a result of major advances in genomics and bioinformatics, including massively parallel sequencing technologies and epitope prediction algorithms.

The author underlines the needs to improve the neoantigens selection criteria to identify a more restricted repertoire of antigens able to induce or amplify a selective response against tumor cells. Among these criteria, the 
choice of mutated antigens derived from CGC genes appears optimal. Two groups of genes are exposed in this classification: (I) genes relevant to cancer: their mutation promotes oncogenic transformation; (II) genes with a strong indication of a role in cancer. Both these groups have the potential to evoke an immune response able to control tumor growth making cancer as a chronic disorder and also to eradicate the disease inducing a complete remission of cancer patients.

Moreover, most OC patients benefit from complete clinical response and several from prolonged survival, however most women will suffer a fatal disease recurrence. Little is known on the mutational load and change in rate that occurs in these different phases of the disease and future studies will necessarily require to answer the question if the disease at different phases continues to express the same or new neoantigens. Such analyses are pivotal when choosing vaccination strategies or lymphocyte transfer.

An optimal lymphocyte priming or boosting can be considered only as a "one side of coin". The immunological elimination of tumor is a complex process with several critical points, such as inhibitory signals on cells and in the microenvironment and lymphocyte trafficking, that need to be targeted or modulated. Beyond the PD-1 and CTLA-4 pathway there are additional suppressive mechanisms that should be modulated in OC patients. A major mechanism involved could be high level of indole-amine2,3-dioxygenase (IDO) that induce $\mathrm{T}$ cell anergy and the development of $T$ cell with regulatory phenotype $(16,17)$. The latter cell population has been demonstrated of pivotal importance in OC (18). Another major problem that cancer immunology is faced with is endothelial modifications that can impact of cell intratumoral migration. In fact, patients with platinum resistant recurrent OC benefit from combination of vasculature-targeted therapies in terms of PFS $(19,20)$. These targeted therapies have demonstrated their efficacy in the improvement of $\mathrm{T}$ cell trafficking and infiltration into the tumor (7).

Still several questions need to be addressed before passing immunological strategies into the clinical practice with a clinically significant change in the history of this disease. Timing of immunological treatment and integration with the several currently adopted therapeutic strategies still need further investigation. A simple example is that it has been clearly demonstrated that primary cytoreduction is able to strongly reduce local and systemic immune suppression. The immunological benefits of surgery are less pronounced when surgery is applied after neoadjuvant chemotherapy or at the time of recurrence (6). It is rationale to believe that this could strongly impact the long-term result of any immunological intervention. Different drug administration strategies such as weekly $v s$. every 3 weeks chemotherapy regimens, intravenous $v s$. intraperitoneal drug administration, adoption of target therapies such as antiangiogenetic monoclonal antibodies or PARP-inhibitors in association with traditional cytotoxic agents or as salvage treatments are only a minority of variables that implicitly will could strongly impact the efficacy of any immunological intervention and that should all be taken into consideration when planning further analyses on patients enrolled into clinical trials. As above mentioned, OC still remains, after several decades of thorough biological and clinical research, the major unsolved problem of gynaecologic oncologists in terms of efficient treatments. However, the last three decades have seen an important change of this disease from rapidly fatal to a chronic condition with an increasingly proportion of women benefiting from prolonged survival. The current goal that thanks to immunotherapy now does seem closer is from a chronic disease to a severe curable condition.

\section{Acknowledgments}

None.

\section{Footnote}

Conflicts of Interest: The authors have no conflicts of interest to declare.

Ethical Statement: The authors are accountable for all aspects of the work in ensuring that questions related to the accuracy or integrity of any part of the work are appropriately investigated and resolved.

\section{References}

1. Zhang L, Conejo-Garcia JR, Katsaros D, et al. Intratumoral $\mathrm{T}$ cells, recurrence, and survival in epithelial ovarian cancer. N Engl J Med 2003;348:203-13.

2. Clarke B, Tinker AV, Lee CH, et al. Intraepithelial T cells and prognosis in ovarian carcinoma: novel associations with stage, tumor type, and BRCA1 loss. Mod Pathol 2009;22:393-402.

3. Sato E, Olson SH, Ahn J, et al. Intraepithelial CD8+ tumor-infiltrating lymphocytes and a high CD8+/ 
regulatory $\mathrm{T}$ cell ratio are associated with favorable prognosis in ovarian cancer. Proc Natl Acad Sci U S A 2005;102:18538-43.

4. Hwang WT, Adams SF, Tahirovic E, et al. Prognostic significance of tumor-infiltrating $\mathrm{T}$ cells in ovarian cancer: a meta-analysis. Gynecol Oncol 2012;124:192-8.

5. Zitvogel L, Apetoh L, Ghiringhelli F, et al. Immunological aspects of cancer chemotherapy. Nat Rev Immunol 2008;8:59-73.

6. Napoletano C, Bellati F, Landi R, et al. Ovarian cancer cytoreduction induces changes in $\mathrm{T}$ cell population subsets reducing immunosuppression. J Cell Mol Med 2010;14:2748-59.

7. Napoletano C, Ruscito I, Bellati F, et al. BevacizumabBased Chemotherapy Triggers Immunological Effects in Responding Multi-Treated Recurrent Ovarian Cancer Patients by Favoring the Recruitment of Effector T Cell Subsets. J Clin Med 2019. doi: 10.3390/jcm8030380.

8. Ding L, Kim HJ, Wang Q, et al. PARP Inhibition Elicits STING-Dependent Antitumor Immunity in Brca1Deficient Ovarian Cancer. Cell Rep 2018;25:2972-80.e5.

9. Siegel RL, Miller KD, Jemal A. Cancer statistics, 2016. CA Cancer J Clin 2016;66:7-30.

10. Odunsi K. Immunotherapy in ovarian cancer. Ann Oncol 2017;28:viii1-7.

11. Antonilli M, Rahimi H, Visconti V, et al. Triple peptide vaccination as consolidation treatment in women affected by ovarian and breast cancer: Clinical and immunological data of a phase I/II clinical trial. Int J Oncol 2016;48:1369-78.

Cite this article as: Napoletano C, Bellati F. Neoantigens from the bench to the bedside: new prospective for ovarian cancer immunotherapy. Ann Transl Med 2019;7(Suppl 8):S305. doi: 10.21037/atm.2019.10.62
12. Alipour S, Zoghi S, Khalili N, et al. Specific immunotherapy in ovarian cancer: a systematic review. Immunotherapy 2016;8:1193-204.

13. Schumacher TN, Robert D. Schreiber. Neoantigens in cancer immunotherapy. Science 2015:348:69-74.

14. Snyder A, Makarov V, Merghoub T, et al. Genetic basis for clinical response to CTLA-4 blockade in melanoma. N Engl J Med 2014;371:2189-99.

15. Liu S, Matsuzaki J, Wei L, et al. Efficient expression of neoantigens-specific T-cell responses in advance human ovarian cancer. J Immunother Cancer 2019;7:156.

16. Tanizaki Y, Kobayashi A, Toujima S, et al. Indoleamine 2,3-dioxygenase promotes peritoneal metastasis of ovarian cancer by inducing an immunosuppressive environment. Cancer Sci 2014;105:966-73.

17. Munn DH, Mellor AL. IDO in the Tumor Microenvironment: Inflammation, Counter-Regulation, and Tolerance.Trends Immunol 2016;37:193-207.

18. Curiel TJ, Coukos G, Zou L, et al. Specific recruitment of regulatory $\mathrm{T}$ cells in ovarian carcinoma fosters immune privilege and predicts reduced survival. Nat Med 2004;10:942-9.

19. Chase DM, Chaplin DJ, Monk BJ. The development and use of vascular targeted therapy in ovarian cancer. Gynecol Oncol 2017;145:393-406.

20. Wu YS, Shui L, Shen D, et al. Bevacizumab combined with chemotherapy for ovarian cancer: an updated systematic review and meta-analysis of randomized controlled trials. Oncotarget 2017;8:10703-13. 